$30 \mid 2003$

Les Aventures de Télémaque. Trois siècles

d'enseignement du français. I.

\title{
Les éditions illustrées des Aventures de Télémaque (1699-XIXe siècle)
}

\section{Claude Bisquerra}

\section{(2) OpenEdition \\ Journals}

Édition électronique

URL : https://journals.openedition.org/dhfles/1472

DOI : $10.4000 /$ dhfles. 1472

ISSN : 2221-4038

Éditeur

Société Internationale pour l'Histoire du Français Langue Étrangère ou Seconde

Édition imprimée

Date de publication : 1 juin 2003

Pagination : 207-219

ISSN : 0992-7654

Référence électronique

Claude Bisquerra, "Les éditions illustrées des Aventures de Télémaque (1699-XIXe siècle) », Documents pour l'histoire du français langue étrangère ou seconde [En ligne], 30 | 2003, mis en ligne le 01 janvier

2012, consulté le 27 mai 2021. URL : http://journals.openedition.org/dhfles/1472 ; DOI : https:// doi.org/10.4000/dhfles.1472

Ce document a été généré automatiquement le 27 mai 2021.

(C) SIHFLES 


\title{
Les éditions illustrées des Aventures de Télémaque (1699-XIXe siècle)
}

\author{
Claude Bisquerra
}

\section{Introduction : l'illustration et le livre}

1 Depuis fort longtemps, texte et image ont été associés pour délivrer des significations parallèles ou compatibles : c'est ce qu'il est convenu d'appeler l'illustration du livre. On ne saurait donc considérer l'écriture et la figure comme deux systèmes radicalement distincts puisque, matériellement, leur union si fréquente n'est possible que par leur figuration commune, le graphisme (Melot 1984).

2 Tout d'abord, qu'entend-on par illustration ? Le terme - un anglicisme - remonte seulement au premier tiers du XIX ${ }^{\mathrm{e}}$ siècle, à 1825 plus exactement. Il se rattache étymologiquement au verbe « illustrer : rendre plus clair, plus intelligible » mais aussi « donner de l'éclat ». L'illustration a d'abord désigné les ornements des anciens manuscrits, puis, lorsque, vers le milieu du XVe siècle le livre imprimé a supplanté le manuscrit, les gravures sur bois insérées dans un texte (Souriau : 1990); dans le sens courant, l'illustration est une image créée expressément pour expliquer ou orner un texte imprimé, livre ou revue, avec lequel elle a un rapport subordonné. Dans le sens étroit, elle ne désigne que les figures incluses dans le texte et met justement l'accent sur ce qui distingue les figures livresques d'autres figures - par exemple des estampes c'est-à-dire leur relation avec le texte et leur insertion dans le tout unitaire qu'est le livre (Robert 1989).

3 Longtemps l'illustration a été considérée comme l'appendice, l'ornement ou la redondance d'un texte et elle n'acquerra sa pleine individualité qu'au XIX siècle. Chronologiquement, elle est seulement ornementale, du livre et non du texte. Mais le plus souvent, elle a une fonction explicative ou interprétative du texte : « La fonction informative de l'image et celle du texte se situent sur deux plans distincts, mais indissociables. L'image peut transmettre une information complémentaire ou supplémentaire, en tout cas différente, plus vite et mieux que le texte» (Robert 1989 : 
s.v. Livre). Les histoires de la bibliophilie ont coutume de considérer le livre illustré comme un lieu d'harmonie mais ce fut le plus souvent un champ clos où se joue une rivalité entre deux systèmes de signes différents.

4 Certains ont dit de l'illustration qu'elle « est un simulacre du contenu textuel qu'elle ne dépasse pas, du moins pas autrement que par les valeurs globales et diffuses de la figuration » (ibid.), d'autres ont tendance à la considérer comme le véhicule sauvage qui autorise autant de sens qu'il y a d'interprètes, face au texte dont le code est contrôlé et qui, par conséquent, peut transmettre " officiellement » du sens (Melot 1984). En faisant preuve d'imagination, l'artiste rejette la transcription littérale du texte. Si certains auteurs ont pu se plaindre de la superficialité de nombreux illustrateurs, comme Balzac qui insistait sur la nécessité que "les dessinateurs lisent le livre " (Zanelli Quarantini 2002)1' d'autres, tels Flaubert ou James, ont refusé catégoriquement les illustrations pour leurs oeuvres, en ce qu'il les considéraient comme une trahison de leur pe nsée (Genette 1987). Quoi qu'il en soit, « l'image est toujours un défi à l'écriture »(Melot $1984: 13)$.

5 Il y a plusieurs manières d'aborder l'illustration : du point de vue de l'histoire du livre, de la bibliophilie, de l'iconographie, de l'esthétique, de la linguistique, de la sémiologie, de la pédagogie, de l'économie et d'autres encore. L'étude des illustrations fait nécessairement appel à la pluridisciplinarité (Gréverand 1983)².

Tout texte, cependant, ne se prête pas à être illustré. On ne peut illustrer un passage d'un livre purement discursif. Il faut des éléments dynamiques. Télémaque est matière à illustration par sa structure même : absence de continuité entre un livre et l'autre, absence de progression des divers épisodes et d'agencement des faits en système, mais successions de tableaux, de " scènes picturales" comme l'ont si souvent mis en évidence les critiques (Fumaroli 1994, Le Brun in Fénelon 1995, Noille-Clauzade 1999). Il ne faut pas oublier, par ailleurs, l'importance que la tradition savante de la pédagogie préceptorale accordait à l'image dans le cadre de l'instruction amusante telle que la préconisait, entre autres, Fénelon qui écrivait dans l'Éducation des filles que les enfants peuvent « apprendre à lire en se jouant » et qu'il faut leur donner « un livre bien relié [...] avec de belles images [...]. Tout ce qui réjouit l'imagination, facilite l'étude " (Fénelon $1804: 51,52$ ). On connait, d'autre part, l'intérêt de Fénelon pour les beauxarts, le rapport qu'il instaure dans différents écrits entre la peinture et l'écriture et vice versa, le rôle qu'il réserve aux arts à travers Mentor au Livre X. Fénelon a-t-il eu connaissance des premières éditions illustrées de son oeuvre ? Télémaque étant en fait le premier roman pour enfants, il y a tout lieu de penser qu'il n'aurait pas désapprouvé les versions illustrées. Mais encore faut-il savoir lesquelles.

\section{Méthodologie, interprétation, résultats}

7 Devant l'étendue des thématiques liées à l'illustration, force est de se limiter à quelques points : tout d'abord, à partir du corpus étudié, établir le pourcentage de livres illustrés : sur l'ensemble des éditions, aux diverses époques, en ce qui concerne les éditions françaises, les éditions étrangères. Ensuite esquisser une typologie formelle des illustrations : déterminer leur place (en frontispice, à l'ouverture de chapitre, au cours du texte etc.), leur nombre à l'intérieur de l'oeuvre, leur ordre, leur récurrence, si elles sont accompagnées ou non de légendes ; puis une typologie contextuelle : quels épisodes ont été illustrés et de quelle manière par rapport au texte. Enfin, chercher à 
relever quels types de messages privilégient les illustrations en fonction du choix des épisodes et des personnages.

\section{1. Établissement du corpus et considérations générales}

8 Le corpus est composé des éditions recensées dans diverses bibliothèques en Italie ${ }^{3}$, sur une période qui s'étend de la première édition en 1699 jusqu'au $\mathrm{XIX}^{\mathrm{e}}$ siècle. Les catalogues et les fichiers des bibliothèques indiquent rarement et mal si le livre contient des illustrations, aussi faut-il recourir aux manuels des bibliophiles pour avoir plus de détails sur les éditions illustrées. Sur les 112 éditions repérées, 16 étaient sans date, 14 non communicables ou manquantes, 9 incomplètes, 1 sans les illustrations indiquées dans l'index. Sur ces 112 éditions, 44 étaient illustrées, toutes en noir et blanc (39\%). Aucune des premières éditions du XVII ${ }^{e}$ siècle n'est illustrée. Brunet fait remonter la première édition illustrée des Aventures de Télémaque à 1705, La Haye, Motjens (Brunet 1861). La première édition illustrée du corpus est de 1712, La Haye, Moetjens, la dernière de 1874, Paravia et Compagnia, réédition identique à celle de 1866 de la même maison d'édition. Pour le XVIII ${ }^{e}$ siècle, 23 éditions illustrées sur un total de 32 (dont 6 non communicables), ce qui fait 23 sur 26 (88\%) ; pour le XIX ${ }^{\text {e }}, 18$ avec illustrations sur les 72 recensées (dont 5 non communicables), ce qui fait 18 sur 67 (27\%). On peut déjà conclure de ce bref examen que le XVIII siècle fournit, comparativement, le plus d'éditions illustrées, ce qui n'a rien d'étonnant puisque ce fut, en France, le siècle d'or du " livre à figures ", surtout après la mort de Louis XIV. Le raffinement qui caractérisait le moindre objet à l'époque s'étend aussi au livre, considéré autant comme livre de lecture que comme livre de chevet en ce sens qu'il " faisait bien » sur le guéridon à la tête du lit. Le livre n'est plus l'enveloppe d'un texte, c'est un moyen de délectation artistique, il devient livre-bibelot de salon. L'illustration joue alors un rôle important dans l'esthétique du livre, elle contribue à sa beautét. Les éditions du XVIII ${ }^{e}$ ont le plus d'illustrations signées de dessinateurs et de graveurs de renom (11 contre 5 au XIX) $)$.

9 La majeure partie des éditions avec illustrations sont en français, 6 en italien dans des éditions imprimées en Italie, 2 en grec imprimées à Venise, 1 bilingue français-anglais imprimée en Angleterre. Les figures sont accompagnées de légendes, sauf dans 6 éditions. Les légendes contribuent à orienter le lecteur. Ce sont en général des phrases concises, résumant l'épisode du texte qui l'a inspiré ou quelquefois des fragments de phrases du texte même (1844, Paris, Belin-Leprieur), " légendes-citations » qui renforcent la participation à la diégèse (Gauthier 1984) ${ }^{5}$. Certaines répètent presque chaque fois le nom de Télémaque (1717, Paris, Estienne), d'autres, où les noms propres abondent (1734, Amsterdam/Rotterdam, Westein, Smith, Houfhout), ont vraisemblablement un but mnémotechnique.

\section{Répartition, nombre, types des illustrations}

10 L'illustration est déterminée, de façon générale, par quelques grands critères, en particulier quant à sa localisation par rapport au texte. L'emplacement de l'image peut être initial, progressif (et parfois itératif) ou terminatif selon que l'illustration intervient au début, au milieu ou à la fin d'un volume ou d'une partie. Se différencient alors les illustrations liminaires, tels le frontispice, la vignette de titre, la lettrine, etc. ; les illustrations $\mathrm{du}$ cours du texte, ponctuées selon un rythme régulier ou non, les 
illustrations en fin de texte comme les culs-de-lampe (Le Men/Caracciolo 1999). Divers facteurs - évolution technique, sociale, économique, tradition éditoriale, mode de chaque époque - déterminent le choix des différents types d'illustrations et leur répartition. C'est ainsi que la technique de la taille-douce, utilisée dans plusieurs de nos éditions, a entraîné « une nouvelle ordonnance du livre car les figures nécessitant un tirage sous presse spécial, ne pouvaient se mêler à l'élément typographique ni s'harmoniser avec lui comme pour les gravures sur bois » (Calivet $1957: 10$ ).

11 Dans nos éditions les figures liminaires sont en nombre réduit ( 7 éditions avec frontispice). Majoritaires, au contraire, les figures au cours du texte : elles sont placées généralement au début de chaque livre, à gauche, en hors-texte, et occupent toute la page ou bonne partie de la page, avec encadrement ou non. Elles ne font pas partie de la séquence des pages du texte (c'est ce qu'on appelle des planches). Ce genre d'illustrations s'apparente à un tableau. Au même titre que les chapitres, elles forment " des coupures régulières, des repos rythmiquement espacés "(Brun 1948: 8). Contrairement à ces illustrations sur toute une page, les vignettes, sans légendes, sont situées au dessus du titre du premier chapitre de chaque livre. L'indélimitation de la forme iconique de la vignette, flottant sur le blanc de la page, l'apparente alors davantage à l'ornement. Les éditions de Venise, Zerletti, 1768 et 1788, offrent un bel exemple de ce mode d'illustrations. Seules les deux éditions de 1852 et 1854, Paris, Havard intercalent des illustrations au cours du texte (pratiquement les mêmes dans les deux éditions).

12 Quant au nombre des figures dans le texte, tout en étant le plus souvent fonction de la division du texte en livres, 10 (1712, La Haye, A. Moetjens), 16 (1710, La Haye, Motjens), 24 (1717, Paris, Estienne), il peut varier arbitrairement : 5 (1716, Paris, Les Associez, 24 livres), 6 (1832, Limoges, Barbou, 24 livres), 8 (1809, Paris, Librairie économique, 24 livres ; 1866, Florence, Paravia et Comp., 24 livres), 9 (1790, Leyde, Wetstein, 10 livres), 12 (1806, Paris, Milan, Scorza et Compagnon, 24 livres), 14 (1807, Turin, Orgeas, 24 livres). La particularité de l'illustration étant de conférer « la dignité d'image à une seule séquence ", en excluant les autres (Zanelli Quarantini 2002 : 23. C'est nous qui traduisons), il est intéressant de voir quelles "bonnes scènes » à illustrer ont été sélectionnées dans les éditions contenant peu de figures.

13 Le frontispice, comme incipit iconographique, joue un rôle particulier : il intervient en " lisière » de l'ouvrage, à la jointure de l'énonciation et de l'énoncé, il annonce et résume le livre, en même temps qu'il appâte le lecteur (Le Men 1979 ; Marin 1990). À la fois prologue, prolepse et synthèse, il peut être comparable à un sommaire synoptique, à " une image de mémoire » qui énonce visuellement par anticipation sur une seule page, les matières que le texte va développer au fil des lignes (Le Men/Caracciolo 1999).

En ce qui concerne les autres types de figures, il arrive bien souvent que des illustrations identiques se trouvent dans des éditions différentes. Ce qui peut être logique quand il s'agit des rééditions d'un même éditeur : ainsi l'édition de 1741, Amsterdam/Rotterdam, Weistein, Hofhout est identique, pour les figures comme pour les légendes, à celle parue quatre ans auparavant en 1737. De même pour les deux éditions de Venise, Zerletti de 1768 et 1788. Mais on assiste parfois à d'évidents recyclages de la part d'autres éditeurs : tel est le cas de l'édition de 1726, Londres, Tonson qui a utilisé les mêmes illustrations que celles de 1717, Paris, Estienne mais en supprimant les signatures du dessinateur Bonnart et du sculpteur Giffrart et en inversant le sens de toutes les figures du second volume; c'est le cas également de celle 
de 1809, Gênes, Gravier qui a " copié » ses 14 illustrations, sur les 24 figurant dans l'édition de 1805, Paris, Le Prieur ; de celle de 1838, Roma, Giunchi et Menicanti, entièrement recyclée sur l'édition de 1809-11, Roma, Perego Savioni. Il y a d'autres exemples. Alors que la plupart du temps les illustrations forment des séries homogènes dues à un même illustrateur, l'édition de 1807, Turin, Orgeas mêle 4 illustrations à 11 autres de facture complètement différente, y compris les caractères des légendes, ce qui nuit à l'unité esthétique du livre. Ces plagiats et autres bizarreries peuvent s'expliquer par les nombreuses difficultés financières que rencontraient les éditeurs et aussi par la vogue des Aventures de Télémaque qui imposait des rythmes accélérés de nouveaux tirages ${ }^{6}$.

\section{Interprétation}

15 Une tentative d'interprétation contextuelle des illustrations doit prendre en compte es domaines de la production/réception de l'oeuvre. Qui décidait le thème, le nombre, la place des illustrations d'un livre? A quels lecteurs était destiné Télémaque ? Autant de questions qui dépassent le cadre de cette recherche. Au XVIII ${ }^{\mathrm{e}}$ siècle, le commerce du livre s'accroît considérablement malgré les réglementations sévères et les charges (Calot et al. 1931). Le livre est le fruit d'une plus étroite collaboration entre dessinateurs, graveurs et libraires-éditeurs. Après la mort de Louis XIV, s'opère une évolution dans l'édition mais aussi dans la condition socioculturelle du lecteur : le public s'élargit, devient plus exigeant, les éditeurs cherchent à accorder le goût de l'artiste à celui du public, hésitent moins devant les frais grâce au système de souscriptions (Bassy 1984). En ce qui concerne la réception de Télémaque, il semble que seules les éditions de 1866 et 74 de Paravia dont on connaît la vocation pédagogique et de Havard, riches en figures intercalées dans le texte, de 1852 et 54 dont la seconde fut publiée dans le « Roman du jour illustré », pouvaient s'adresser plus particulièrement à de jeunes lecteurs.

16 Pour ce qui est de la méthodologie adoptée, faute d'une étude diachronique et synchronique complète, il a d'abord été établi la confrontation des illustrations de toutes les éditions pour déterminer la fréquence des thèmes et leurs occurrences respectives, puis la confrontation des illustrations d'un même thème dans des éditions différentes pour en relever et en analyser les variantes.

17 L'on constate en premier lieu - avec les restrictions qui s'imposent quand on sait qu'un certain nombre des éditions examinées ne nous sont pas parvenues intégralement que l'épisode le plus souvent illustré est celui de " la descente de Télémaque aux enfers», aussitôt suivi, presque ex-aequo, par ceux de "Mentor sortant de Salente et proposant la paix » et "Télémaque tuant Adraste ». Très près derrière, "Télémaque et Mentor abordent l'île de Calypso", puis "Télémaque jeté à la mer du haut d'un rocher ». Tous ces épisodes ont de fortes occurrences. Viennent ensuite, toujours par ordre dégressif, « Télémaque arrive à Salente ", épisode autant représenté que la scène de chasse où Télémaque protège Antiope contre le sanglier blessé, puis « le commerce de Tyr et Narbal ", "les Champs Élysées », "le sacrifice d'Idoménée ", "la rencontre dans le désert avec Termosiris ", "Philocles désarmant ses assassin ", etc. Parmi les illustrations qui ont le moins d'occurrences : «Pigmalion empoisonné par Astarbé », « Hercule au bûcher », «Idoménée fait chanter sa fille devant Télémaque ». 


\section{Analyse pour l'ensemble du corpus}

Sur la plupart des illustrations figure Télémaque, et souvent sans Mentor. Il est incontestablement le héros-modèle sur lequel doit se focaliser l'attention ${ }^{7}$. Cette focalisation est accentuée par la répétition, dans certaines légendes, du nom de Télémaque, au début ou au cours de la phrase. Comme dans le texte, les illustrations alternent moments d'action violente et moments de repos : l'on a ainsi d'une part, Télémaque presque toujours représenté en guerrier, de nombreuses scènes sur fond de bataille ou de soldats en armes, trois épisodes montrant une mise à mort ; d'autre part, le calme avec le cadre idyllique des Champs Élysées, l'Académie des beaux arts, Télémaque contant ses aventures à Calypso, les dieux marins chantant autour du vaisseau de Télémaque. À travers les illustrations à forte occurrence, Télémaque, plus souvent acteur que spectateur, incarne le symbole à la fois d'un pouvoir détenu par une seule personne et d'un chef entreprenant, courageux, et juste. D'autres messages transmis par ces illustrations pourraient être que la vie, plus particulièrement celle d'un futur roi, est la lutte continuelle d'un vainqueur et encore qu'il faut tenir compte des conseils et de l'expérience des autres comme le suggèrent les figures respectables de Mentor, Narbal, Termosiris et Arcésius qui accompagnent le héros. S'ajoutent des leçons de sagesse pratique (fuir les dangers quand ils concernent la sphère des sentiments), de noblesse de sentiments (amour filial, amitié), de politique (nécessité de la paix/élimination de la traîtrise), d'économie et de religion (opposition vrai Dieu/faux dieux). Autant de thèmes hiérarchisés du texte que les illustrations reflètent fidèlement.

\section{Variantes d'une même illustration dans des éditions différentes}

\section{- frontispice}

Ceux des éditions 1712, La Haye, Moetjens, (fig. 2), 1717, Paris, Estienne (fig. 3), 1805, Paris, Le Prieur (fig. 4), 1790, s'appuyant sur les normes d'un système géométrique simple - partition et opposition selon les médianes horizontales ou verticales, liaison sur la diagonale, inclusion/exclusion, premier plan/fond (Bassy 1984) - soulignent l'importance de Minerve menant Télémaque sur le sentier de la vertu, finalité du héros, symbolisée par le temple. Plus élaborés dans le dessin et plus riches de détails, ceux de 1799, Venezia, Curti (fig. 5) et de la très belle édition de 1734, Amsterdam/Rotterdam, Wetstein, Hofhout (fig. 6), celui-ci accompagné d'une longue légende récapitulative, montrent le héros encore tout attiré par les plaisirs terrestres, tournant le dos au temple.

\section{- Télémaque aux enfers}

Parfois la figure représente le fils d'Ulysse s'apprêtant à entrer dans l'antre, encore sur le seuil du monde des vivants, dans une attitude statique ou marchant vers la sombre entrée ; ou bien sur le point de monter dans la barque de Caron, ou encore devant Pluton, entouré de damnés, de monstres et de flammes, toujours en guerrier et parfois accompagné de Minerve. Au delà de simples variantes stylistiques de la part des artistes, on peut voir dans cette diversité de représentation la mise en relief d'un moment précis de l'engagement de Télémaque : sur le seuil de l'enfer, seul, alors que les deux guerriers se sont éloignés (fig. 7, 8, 9), c'est l'instant de la prise de décision avant 
l'action. Un pas de plus est franchi avec la barque de Caron (fig. 10, 11), zone intermédiaire entre le monde des vivants et celui des morts où, si la détermination est irrévocable, la confrontation avec l'enfer n'est pas encore effective. Télémaque arrivé en enfer (fig. 12,13) évoque la représentation matérielle du lieu et l'horreur de la punition des fautes de l'humanité tout entière ; représenté seul devant le trône de Pluton (fig. 14), il incarne alors le jugement individuel de chacun. Quand elle est présente aux côtés de Télémaque, Minerve est l'assurance de la protection divine (fig. 15).

\section{- Télémaque précipité dans la mer}

21 Trois illustrations montrent en premier plan les nymphes incendiant le vaisseau et, indistinctement en arrière plan, deux silhouettes tombant dans le vide. Le thème de la vengeance lié à la douleur d'amour de Calypso est prédominant. Les autres, plus nombreuses, focalisent l'instant où Télémaque tombant en chute libre, la tête la première, les gestes désordonnés, avec, plus ou moins marquée, une impression de terreur sur le visage, est sur le point d'être englouti par les flots (fig. 16, 17, 18). Mentor, quant à lui, touche encore la terre d'un pied ou est représenté très près de la falaise. Il donne l'impression qu'il est suspendu dans les airs, défiant la loi de la gravité et, contrairement à Télémaque, en train de voler (fig. 19, 20). Seul Télémaque paraît être concerné par la chute, par la chute dans l'abîme selon la symbolique de l'élévation et de l'abaissement de l'âme à travers un axe de la verticalité qui va du haut - c'est le cas de Mentor - au plus profond - c'est le cas de Télémaque. Mentor, symbole de l'élan vers les hauteurs, échappe à la chute tandis que le poids de la faute entraîne Télémaque vers l'abîme du néant ${ }^{8}$.

\section{- Illustrations d'ouverture/de clôture du texte}

Pour celles-ci, contrairement aux autres, il ne s'agit plus de variantes sur un même thème mais de choix de séquences de statuts inégaux du récit.

La première illustration qui ouvre le texte offre plusieurs exemples sur le thème de la rencontre avec Calypso : les unes intensifient le naufrage en montrant Télémaque et Mentor, ballottés par les flots en furie, agrippés à des débris, sous un ciel d'orage traversé d'éclairs (fig. 21); d'autres mettent au premier plan le moment de la rencontre de Télémaque avec Calypso, alors qu'il est déjà sur le rivage et que le naufrage est plus ou moins évoqué dans le lointain (fig. 22, 23, 24), ou reçu par Calypso dans sa grotte (fig. 25) ; d'autres encore réunissent toujours plusieurs moments du récit mais anticipent le rôle de la nymphe dans la suite de l'histoire, avec Télémaque-Calypso devant un arbre dont deux branches entremêlées symbolisent l'union (fig. 26), ou formant déjà un couple (fig. 27). Pour l'illustration finale nous trouvons trois alternatives : la scène de la chasse dans 4 éditions seulement (London 1742, Limoges, Barbou 1832, Paris, Mallet et Cie 1840, Torino, Fontana, Pomba e Comp. 1842, Venezia, Curti 1799) (fig. 28), les retrouvailles de Télémaque avec Ulysse, épisode à peine plus fréquemment illustré que celui représentant Minerve qui quitte la figure de Mentor et se sépare de Télémaque. Bien souvent, la première édition illustrée d'une oeuvre détermine les stéréotypes des éditions successives ${ }^{9}$. Malheureusement, il ne nous a pas été possible d'examiner les 7 figures de la première édition illustrée selon Brunet (1705, La Haye, Motjens $)^{10}$. Les premières éditions avec illustrations que nous avons pu consulter, 1710, La Haye, 
Motjens (Bibliothèque Nationale, Paris) (fig. 29) et 1712, La Haye, Moetjens, (Archiginnasio, Bologne) (fig. 30), contiennent le second schéma, Minerve quittant la figure de Mentor. En revanche, l'édition de 1717 de Paris, Delaulne, reprise à la même date par Estienne, à l'origine de nombreuses éditions successives françaises et étrangères au XVIIIe siècle (Le Brun 1995 : 427), montrent Télémaque retrouvant Ulysse (fig. 31). Si l'on s'en tient à une interprétation strictement littérale, les retrouvailles de Télémaque et de son père réduisent les Aventures à une saga familiale qui se termine sur une fin heureuse, tout empreinte de sentimentalisme. Au terme de ses aventures, Télémaque réintègre l'univers protecteur de la famille dominé par la puissante figure paternelle à laquelle il sera constamment confronté, dans la réalité cette fois. Quelques illustrations insistent sur le caractère intime et familial de la scène en situant les personnages à l'intérieur de l'espace clos d'une pièce où Ulysse est en train de se restaurer ou tient Télémaque affectueusement embrassé (fig. 31, 32, 33). Si l'on interprète l'illustration dans une vision chrétienne, Télémaque, au terme de son parcours terrestre, est revenu à la maison du père, devant lequel il se tient respectueusement agenouillé (fig. 31).

Dans l'autre schéma au contraire, le livre se termine sur l'image de Minerve ayant repris sa forme divine. Une lecture païenne représente, sur fond de décor sylvestre, la simple métamorphose de Mentor en Minerve, à peine soulevée au dessus du sol, Télémaque debout devant elle (fig. 35) ; dans une autre optique, tout concourt, pour peu qu'on sache décrypter les symboles de l'image, à transmettre un message chrétien. André Blanc (Blanc 1979) a comparé cette scène à la transfiguration du Christ et à son assomption, en se référant aux phrases de Fénelon qui parlent de la jeunesse et de la « majesté simple » du visage de Minerve, de l'éclat de ses vêtements et de l'éblouissement de Télémaque. Télémaque est alors agenouillé dans une attitude d'extase et de vénération devant le père, mais le Père avec une majuscule (fig. 36) ${ }^{11}$. Du plan du terrestre ou du mythe païen, nous sommes passés au plan du divin. Comme les apôtres, Télémaque doit s'habituer à être privé de la douceur de la présence de son Mentor, il est temps maintenant qu'il apprenne à marcher tout seul. Toutefois, bien que différents, ces deux types d'illustrations peuvent être lus comme les deux représentations d'une même interprétation catholique de la même histoire : l'une met en relief l'aspect humain du parcours de Télémaque avec le retour à la maison, l'autre l'aspect divin, l'accès au ciel, avec l'accompagnant qui se transfigure en la divinité.

\section{Conclusion}

La tentative d'établir un corpus - sur une période volontairement limitée à deux siècles - des éditions illustrées des Aventures de Télémaque recensées dans diverses bibliothèques italiennes a permis de retracer un aperçu de l'évolution historique des illustrations en déterminant leurs typologies et en mettant en évidence la présence de thèmes dominants et de variantes se référant à des valeurs morales et politiques. Les premiers résultats partiels montrent que les illustrations, tout en étant esthétiquement traitées à chaque époque de manière différentes selon les goûts et la personnalité de l'artiste qui s'affirme toujours davantage au cours du XVIII ${ }^{e}$ siècle, reflètent les idéologies qui sous-tendent l'oeuvre et ses interprétations traditionnelles. Probablement conséquence des aléas de la tradition éditoriale, cette constante ne fait que conforter, néanmoins, une lecture codifiée de l'oeuvre, aux finalités précises. Il va 
de soi que pour être significative cette analyse devrait s'étendre non seulement à l'ensemble des éditions illustrées des Aventures mais encore s'appuyer sur les résultats des études d'autres oeuvres illustrées ayant eu une diffusion et un succès similaires, sur la même période examinée. Alors qu'au moment de la lecture du texte seul le lecteur jouit d'une relative liberté dans ses choix interprétatifs, les illustrations, immanquablement arbitraires, le guident sur le chemin balisé de l'interprétation intermédiaire de l'artiste et de l'éditeur, s'interposant avec plus ou moins d'insistance selon qu'elles apparaissent sporadiquement dans l'oeuvre ou en scandent régulièrement le découpage en livres. Faut-il préférer les premières, au risque de frustrer notre émotion esthétique ou les secondes, au risque de provoquer notre passivité interprétative ? Comme l'a justement écrit Claudel à propos du livre illustré : «l'union du dessin et de la typographie comporte bien des combinaisons, je ne sais si aucune d'elle n'a jamais été complètement satisfaisante » (Claudel 1925 : 128. Cité par Le Men 1995 : 229). Contrairement au critique dont la démarche s'accompagne d'un effet pléthorique de la visualisation de l'imaginaire, le lecteur, possesseur le plus souvent d'un unique exemplaire illustré de Télémaque, a tout loisir, au fil de ses lectures réitérées, sur la sollicitation de quelques images, de laisser se déployer librement son imagination pour créer sa propre représentation visuelle de l'oeuvre.

\section{Bibliographie}

\section{Sources manuscrites}

Rome : Biblioteca American Academy, Biblioteca Angelica, Biblioteca Apostolica Vaticana, Biblioteca Casanatense, Biblioteca Centrale Nazionale, Biblioteca della Fondazione Marco Besso, Biblioteca della Libera Università Santissima Assunta, Biblioteca universitaria Alessandrina, Biblioteca universitaria Angelo Monteverdi, Biblioteca universitaria di Lingue e Letterature Moderne Straniere, École française.

L'Aquila : Archivio di Stato, Biblioteca del Centro celestiniano, Biblioteca del convento di S. Chiara, Biblioteca del convento di S. Giuliano, Biblioteca della Facoltà di Lettere e Filosofia, Biblioteca provinciale Salvatore Tommasi.

Bologne : Biblioteca Comunale dell'Archiginnasio, Biblioteca del Dipartimento di Lingue e Letterature Straniere Moderne, Biblioteca universitaria.

\section{Sources imprimées}

BARTHES, Roland (1964), « Rhétorique de l'image », Communications 4, repris dans L'Obvie et l'Obtus, Paris, Seuil, 1982. 
BASSY, Alain-Marie (1984), « Le texte et l'image », in Martin \& Chartier (dir).

BLANC, André (1979), « Fonction de la référence mythologique dans Télémaque », XVII ${ }^{e}$ siècle 125, octobre-décembre, 373-388.

BOISSAIS, Maurice, DELEPLANQUE, Jacques (1948), Le livre à gravures au XVIII ${ }^{e}$ siècle, Paris, Gründ. BRUN, Robert (1948), Le livre français, Paris, PUF.

BRUNET, Jean Charles (1861), Manuel du libraire et de l'auteur de livres, Paris, Firmin Didot frères, fils et Cie.

CALIVET, Diane (1957), L'illustration de la poésie et du roman français au XVII'siècle, Paris, PUF.

CALOT, Frantz, MICHON, Louis-Marie, ANGOULVENT, Paul (1931), L'art du livre en France des origines à nos jours, Paris, Delagrave.

CARACCIOLO M. Teresa, LE MEN Ségolène dir. (1999), L'illustration. Essais d'iconographie. Actes du séminaire CNRS, Paris, 1993-94, Paris, Klincksieck.

CLAUDEL, Paul (1925), « La philosophie du Livre », conférence faite à l'Exposition du Livre de Florence in Réflexions sur la poésie, Paris, Gallimard (1969).

COHEN, Henri (1912), Guide de l'amateur de livres à gravures du XVIII siècle, 6e éd., Paris, A. Rouquette.

Encyclopædia universalis (1980), Paris, Encyclopædia universalis.

FÉNELON (1804), L'éducation des filles, Lyon, Leroy.

-----(1995), Les aventures de Télémaque, édition présentée, établie et annotée par de J. Le Brun, Paris, Gallimard, « Folio classique ».

FUMAROLI, Marc (1994), "Visions et prière : La Rencontre de Jésus et du Baptiste du Guide ", in L'École du silence. Le sentiment des images au XVII siècle, Paris, Flammarion.

GAUTHIER, Guy (1984), «Image et texte. Le récit sous le récit », Langages 75, septembre 1984, 9-21.

GENETTE, Gérard (1982), Palimpsestes : la littérature au second degré, Paris, Seuil.

------(1987), Seuils, Paris, Seuil.

GOULEMOT, Jean-Marie (1984), « Nouveautés : les utopies » in MARTIN / CHARTIER dir.

GRÉVERAND, Gérard (1983), « L'illustration des Fables de La Fontaine. 1668-1980 » in Iconographie et littérature. D'un art à l'autre, Centre d'études et de recherche d'histoire des idées et de la sensibilité, Paris, PUF, 81-96.

JAKOBSON, Roman (1963), Aspects linguistiques de la traduction, Paris, Éd. De Minuit.

LE MEN, Ségolène (1979) « Quant au livre illustré... », Revue de l'art 44, 85-111.

------(1995), « La question de l'illustration » in R. Chartier (dir.), Histoires de la lecture. Un bilan de recherches, Paris, IMEC éd., Édition de la maison des Sciences de l'Homme.

MACHET, Anne (1984), « Le marché italien », in Martin \& Chartier (dir).

MARIN, Louis (1990), « Préface-image, le frontispice des Contes de Perrault », Europe 739-740 :

Charles Perrault, novembre-décembre, 114-121.

MARTIN, Henri-Jean (1969), Livre, pouvoirs et société à Paris au XVII siècle (1598-1701), Paris-Genève, Droz. 
MARTIN, Henri-Jean, CHARTIER, Roger (dir.) (1984), Histoire de l'édition française. Le Livre triomphant 1660-1830, Paris, Promodis, t. II.

MELOT, Michel (1984), L'illustration. Histoire d'un art, Genève, Skira.

MONTANDON, Alain dir. (1990), Iconotextes, Clermont-Ferrand, Berlin, Orphys.

NOILLE-CLAUZADE, Christine (1999), « La morale du Télémaque : pour une poétique platonicienne de la fable ", Revue des sciences humaines 254, avriljuin : Morale et fictions aux XVII et XVIII ${ }^{e}$ siècles, 85-106.

PAPASOGLI, Benedetta (2000), « Espace intérieur et vie spirituelle de Fénelon » XVII ${ }^{e}$ siècle 206, janvier-mars, 57-72.

PERROT, Jean-Claude (1984), « Nouveautés : l'économie politique et ses livres », in Martin \& Chartier (dir).

ROBERT (1989), Le grand Robert de la langue française. Dictionnaire alphabétique et analogique de la langue française, Paris, Le Robert.

SOURIAU, Étienne (1990), Vocabulaire d'esthétique, Paris, PUF.

TCHEMERZINE, Avdenir (1977), Éditions originales et rares. XVe-XVIII siècle, Paris, Hermann, t. III.

WEIGERT, Roger-Armand (1930-1962), I.F.F., Inventaire du fonds français. Graveurs du XVIII ${ }^{e}$ siècle, Paris, Bibliothèque Nationale.

ZANELLI QUARANTINI, Franca (2002), Il testo e l'immagine. Indagine sulle " gravures " d'accompagnamento a Manon Lescault, La Nouvelle Héloïse, Les Liaisons dangereuses, Bologna, CLUEB.

\section{NOTES}

1. Balzac, Correspondance, 1960, IV, cité par Zanelli-Quarantini $2002: 21$.

2. Chacune des disciplines étudiera l'image selon ses propres exigences : pour l'historien du livre, l'illustration fait partie de l'objet qu'il étudie mais elle apparaît en marge de son enquête ; pour l'historien des images, les perspectives s'inversent. Quant au rapport de l'image - ou de la « figuration » - aux textes, il a été largement traité, dans la théorie et la pratique, par la recherche iconologique. Sur la nature de la relation image-texte plusieurs thèses et hypothèses ont été aussi émises : pour Jackobson, l'illustration rentre dans une esthétique de la traduction comme procédure de reformulation d'une part et de traduction intersémiotique d'autre part (Jakobson 1963), Barthes a parlé d'« ancrage » ou de « relais » (Barthes 1964), Genette a introduit la notion de « paratexte » (Genette 1982), plus récemment a été forgé le terme « iconotexte » (Montandon 1990) et l'on traite toujours de l'opposition traditionnelle entre illustration " littérale», interprétative ou typographique (Caracciolo/Le Men 1999).

3. Principalement de Rome et l'Aquila pour notre propre dépouillement ; ont été incorporées les éditions illustrées des données des bibliothèques de Bologne fournies par Nadia Minerva ; ont été exclues les éditions d'un même éditeur, ayant la même date, c'est-à-dire les " doubles ", qui se trouvaient dans des bibliothèques différentes, ainsi que toutes les éditions sans date.

4. Certains livres ont même dû leur valeur davantage à leurs illustrations qu'à leur texte.

5. Tout comme l'absence de citation, mais contrairement à la "légende-commentaire » qui accentue au contraire la prise de distance, l'effet documentaire (Gauthier 1984).

6. 58 rééditions de Télémaque au XVIIIe siècle d'après le catalogue des imprimés de la Bibliothèque Nationale de Paris (Goulemot 1984). 
7. Même si l'illustration de Télémaque transporté de colère, jetant sa lance contre Nippias qui lui a enlevé ses captifs est anti-héroïque (fig. 1), relève d'une interprétation très personnelle du texte et de l'illustration.

8. L'idée de la symbolique de l'élévation est empruntée à Papasogli 2000.

9. C'est ainsi, par exemple, que la face grimaçante de Quasimodo de la première édition illustrée de Notre-Dame de Paris de Victor Hugo se retrouve encore dans une bande dessinée contemporaine (Le Men 1995).

10. Le volume, conservé à la Bibliothèque Nationale de Paris, était en cours de reproduction.

11. Sur une illustration, le mont représenté pourrait être le mont Tabor, avec les disciples (fig. 29).

\section{RÉSUMÉS}

Plusieurs éditions des Aventures de Télémaque sont illustrées. Cette étude s'est appliquée à faire un recensement des éditions illustrées des diverses bibliothèques italiennes (Rome, L'Aquila, Bologne) de 1699 au XIX ${ }^{\mathrm{e}}$ siècle, dans le but d'en établir le pourcentage selon les époques, d'esquisser une typologie formelle et contextuelle des illustrations, d'en définir les messages, d'en relever les thèmes et leur fréquence, de confronter différentes illustrations d'un même thème pour mettre en évidence et analyser leurs variantes.

Several editions of Telemacus are illustrated. This study attempts to create a census of the illustrated editions present in various Italian libraries (Rome, L'Aquila, Bologna) from 1699 to the $19^{\text {th }}$ century. The aim of this study is to a) evaluate the percentage of illustrated editions in different centuries, b) outline the formal and contextual typologies of the illustrations, $c$ ) decipher possible messages present in the illustrations, $d$ ) point out existing themes as well as the frequency of their occurrence, $c$ ) compare the different illustrations pertaining to the same theme, $f$ ) point out variants and analyse them.

\section{INDEX}

Mots-clés : 1699-XIXe siècle, Aventures de Télémaque, illustrations

Keywords : 1699-19th century, illustrated editions, Telemacus

\section{AUTEUR \\ CLAUDE BISQUERRA \\ Università dell'Aquila}

